\title{
Implantación de una Consulta de Enfermería Vascular en un hospital de referencia
}

\author{
Rosa Casado Fernández ${ }^{1}$, Esperanza Zuriguel-Pérez ${ }^{2}$, Isabel Zafra Izquierdo ${ }^{3}$, Montserrat Candel Alonso ${ }^{4}$, José María \\ Escribano Ferrer ${ }^{5}$, Neus Pelay Panés ${ }^{6}$, Sergi Bellmunt Montoya ${ }^{7}$, Roser Lara Fernández ${ }^{8}$ \\ ${ }^{1}$ Enfermera del Servicio de Angiología y Cirugía Vascular, Hospital Universitari Vall d'Hebron de Barcelona. ORCID \\ 0000-0003-0117-2632. \\ ${ }^{2}$ Coordinadora de Investigación en Enfermería, Hospital Universitari Vall d’Hebron de Barcelona. ORCID 000-0002- \\ 0622-8423. \\ 3, 4 Enfermera del Servicio de Angiología y Cirugía Vascular, Hospital Universitari Vall d’Hebron de Barcelona \\ ${ }^{5}$ Médico Adjunto del Servicio de Angiología y Cirugía Vascular, Hospital Universitari Vall d’Hebron de Barcelona. \\ ${ }^{6}$ Supervisora de enfermería de las Consultas Externas del Hospital Universitari Vall d'Hebron de Barcelona. \\ ${ }^{7}$ Jefe de Servicio de Angiología y Cirugía Vascular, Hospital Universitari Vall d'Hebron de Barcelona. \\ ${ }^{8}$ Adjunta de la Dirección de Enfermería. Atención ambulatoria del Hospital Universitari Vall d’Hebron de Barcelona.
}

\section{Correspondencia}

Rosa Casado Fernández

Correo electrónico: rcasado@vhebron.net

Recibido: $10 / 06 / 2020$

Aceptado: $27 / 06 / 2020$

\section{RESUMEN}

El objetivo de este trabajo fue describir la puesta en marcha y organización de la Consulta de Enfermería Vascular del Hospital Universitario Vall d'Hebron, detallar la actividad realizada durante sus primeros seis meses de existencia, analizar los obstáculos que dificultan la práctica profesional autónoma de las enfermeras que trabajan en esa consulta y proponer soluciones.

Se evaluó el número de visitas de la consulta, los diagnósticos médicos y enfermeros de los pacientes, las prestaciones enfermeras ligadas a la visita y el tipo de heridas que presentaban los pacientes. Se atendieron 153 pacientes y la mayoría de prestaciones enfermeras registradas fueron: "Educación sanitaria" y "Cuidados de heridas y otras lesiones".

El principal obstáculo detectado hasta la fecha es de índole tecnológico, ya que la aplicación informática utilizada en las consultas externas del hospital, lejos de facilitar el rol autónomo enfermero, lo dificulta.

La Consulta de Enfermería Vascular potencia la autonomía de la práctica profesional y favorece la atención personalizada a los pacientes de las consultas externas del Servicio de Angiología y Cirugía Vascular. Para que las enfermeras de esta consulta puedan desarrollar todo su potencial, y para que los resultados de sus actuaciones sean mesurables y visibles, es necesario implementar sistemas de información asistencial que sean compatibles con una herramienta metodológica tan básica para la profesión como es el proceso enfermero, y que permitan registrar los datos derivados de la valoración del paciente y de toda la actividad realizada mediante vocabularios normalizados. Dentro de los vocabularios empleados en nuestro entorno, pensamos que la terminología ATIC sería la opción más conveniente.

\section{PALABRAS CLAVE}

enfermería de consulta; práctica profesional; sistemas de información en hospital; informática aplicada a la enfermería; terminología normalizada de enfermería.

\section{SUMMARY}

The objective of this work was to describe the start-up and organization of the Vascular Nursing Office of the Vall d'Hebron University Hospital, to detail the activity carried out during its first six months of existence, to analyze the obstacles that hinder the autonomous professional practice of the nurses working in that office and to propose solutions.

The number of visits for consultation, the medical and nursing diagnoses of the patients, the nursing interventions and the type of wounds presented by the patients were evaluated. 153 patients were treated and the majority of registered nursing interventions were: "Health education" and "Care of wounds and other injuries".

The main obstacle detected to date is of a technological nature, since the computer application used in outpatient hospital consultations, far from facilitating the autonomous role of nurses, makes it difficult. 
The Vascular Nursing Consultation enhances the autonomy of professional practice and favors personalized attention in outpatient consultations of the Angiology and Vascular Surgery Service. For the nurses in this consultation to achieve their full potential, and for the results of their actions to be measurable and visible, it is necessary to implement healthcare information systems that are compatible with such a basic methodological tool for the profession as the nursing process, and that allow recording the data derived from the assessment of the patient and all the activity carried out by using standardized vocabularies. Within the vocabularies used in our environment, we think that the ATIC terminology would be the most convenient option.

\section{KEYWORDS}

office nursing; professional practice; hospital information systems; nursing informatics; standardized nursing terminology.

\section{Introducción}

Una consulta de enfermería es un servicio técnico, científico, libre y autónomo que se otorga por profesionales de enfermería, ya sea en el ámbito público o privado, dentro y fuera de los hospitales y en todos los niveles de atención sanitaria. Su propósito principal es promover, habilitar, incluir, prevenir, detectar, resolver necesidades y problemas de salud del individuo, familia y comunidad en todas las etapas de la vida (1). Para los profesionales, estas consultas, funcionan como la puerta de entrada hacia la autonomía profesional, la práctica independiente y el reconocimiento social (1). Entre otros atributos, en estas consultas se aplica una metodología científica (PAE) y se implementan las tecnologías de la información y la comunicación (1).

La evidencia destaca que el manejo de heridas es predominantemente una disciplina dirigida por enfermeras y que, gracias a su formación, están capacitadas para prevenir, educar, diagnosticar, tratar y curar tanto a los pacientes como a sus familiares (2). Las denominadas unidades de heridas crónicas son estructuras organizativas diseñadas para proporcionar cuidados integrales a las personas con heridas crónicas, mediante la utilización de un modelo metodológico preestablecido $(3,4)$.

El aumento de la esperanza de vida se corresponde con un incremento de las enfermedades crónicas y un número creciente de pacientes pluripatológicos, de tal forma que una buena coordinación entre Atención Hospitalaria y Atención Primaria es indispensable (5)

El mejor modelo de relación transversal en el cuidado de las heridas crónicas es, sin duda, el equipo multidisciplinar, y se ha demostrado que estas heridas curan más eficazmente cuando el paciente es atendido de manera holística por un equipo multidisciplinar que centre su atención en la persona y no sólo en la herida (6).

Una de las características definitorias de una disciplina profesional es la de utilizar una metodología propia para resolver los problemas de su competencia. La denominada metodología científica, al aplicarse a nuestro ámbito, ha recibido el nombre de proceso enfermero (PE) (7). El PE es un método sistemático de brindar cuidados humanistas eficientes centrados en el logro de resultados esperados (8), y los planes de cuidados son la expresión escrita de su aplicación. El uso de registros médicos electrónicos y sistemas de información en todos los niveles de las agencias de salud está ahora muy extendido en todo el mundo. Con el fin de optimizar la eficiencia de estos registros y sistemas y facilitar el intercambio de información entre profesionales e instituciones, deben basarse en vocabularios controlados (9).

La generación de vocabularios enfermeros se vincula al desarrollo teórico para la identificación, la definición y la clasificación de los conceptos disciplinares (9). El lenguaje desempeña un papel importante en la definición de lo que hacen las enfermeras y por qué lo hacen. Los vocabularios de enfermería controlados se pueden implementar como terminologías de interfase en el punto de atención y como terminologías administrativas para recuperar datos clínicos de enfermería con el fin de apoyar la toma de decisiones (9). La Taxonomía Internacional de la Asociación Norteamericana de Diagnósticos de Enfermería (NANDA) (10) y las clasificaciones Nursing Interventios Classification (NIC) (11) y Nursing Outcomes Classification (NOC) (12) del University of lowa College of Nursing son los vocabularios normalizados más extendidos. NANDA, NOC y NIC son de uso obligatorio en el sistema sanitario español según el Real Decreto 1093/2010 del 3 de septiembre. En el Sistema Català de la Salut se emplea la terminología ATIC (siglas del acrónimo Arquitectura, Terminología, Enfermería y Conocimiento en catalán), que es un vocabulario estandarizado, orientado a conceptos, multiaxial, de alta especificidad, de interfase y validado (13).

En el Hospital Universitario Vall d'Hebron (HUVH) de Barcelona existen consultas de enfermería de las ya conocidas en el ámbito hospitalario nacional, y el hospital cuenta también con una Unidad de Heridas Crónicas en la que la enfermera atiende a pacientes de cualquier especialidad, médica o quirúrgica. En este caso, esa estructura funciona como una unidad de atención especializada y tiene como objetivo proporcionar cuidados integrales a personas con heridas crónicas. En el hospital también existe una Unidad de Pie Diabético que está capacitada para tratar las úlceras complicadas, las lesiones recidivantes y pies con elevado riesgo de ulceración, frecuentemente con neuropatías y deformidades severas. En la Unidad de Pie Diabético la enfermera está presente en todas las áreas de actuación y colabora en la implantación de las guías de práctica clínica para la prevención y manejo de esta patología. 
En las Consultas Externas de ACV del HUVH siempre ha habido enfermeras que desempeñaban un rol básicamente colaborativo realizando pruebas diagnósticas, curas de heridas y ayudando a los cirujanos a realizar esclerosis venosas. La dirección de enfermería del HUVH está desarrollando y desplegando nuevos perfiles enfermeros, con rol autónomo, enfocados hacia la atención a la cronicidad y por este motivo el servicio de ACV y la Dirección Asistencial del hospital facilitaron la puesta en marcha de la Consulta de Enfermería Vascular (CEV), como un elemento más en el equipo de dicho servicio, con los objetivos de mejorar los cuidados que reciben los pacientes derivados a las consultas externas de esta especialidad desde atención primaria, desde la unidad de hospitalización de ACV y desde otros servicios del hospital, y de garantizar la continuidad de los cuidados.

En las unidades de hospitalización de muchos hospitales las enfermeras utilizan sistemas informáticos que les facilitan el trabajo y les permiten registrar los datos que reflejan toda su actividad asistencial, tanto la valoración de los pacientes como los planes de cuidados, la administración de medicamentos, los comentarios o los informes de continuidad de cuidados. En las consultas externas del HUVH, las enfermeras solo tienen acceso al sistema gestión administrativa de pacientes (SAP), que está conectado con HC3 (14) (una plataforma que hace posible acceder a la información clínica de los pacientes tratados en los diferentes centros sanitarios de Cataluña) y que sirve para realizar la gestión administrativa y clínica del paciente. A nivel administrativo, SAP es una herramienta muy versátil, pero a nivel clínico solo permite que las enfermeras registren "prestaciones de cuidados", un grupo de 20 actuaciones preestablecidas pero que no están bien definidas ni estandarizadas (Tabla 1). SAP tampoco está diseñado para trabajar con planes de cuidados.

Tabla 1: Prestaciones incluidas en SAP

\begin{tabular}{|c|c|}
\hline \multicolumn{2}{|r|}{ Prestaciones de enfermería } \\
\hline $\begin{array}{l}\text { Prestaciones } \\
\text { realizables }\end{array}$ & \\
\hline 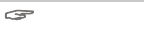 & Visita para cuidados de enfermería \\
\hline$\sigma$ & Cuidados de alimentación y nutrición \\
\hline$\sigma$ & Cuidados de bienestar \\
\hline$\sigma$ & Cuidados de catéteres \\
\hline$\sigma$ & Circuitos \\
\hline$\sigma$ & Cuidados de comunicación e interacción \\
\hline$\sigma$ & Control de parámetros y monitorización \\
\hline$\sigma$ & Cuidados asociados a creencias y valores \\
\hline$\sigma$ & Cuidados de heridas y otras lesiones \\
\hline 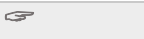 & Educación sanitaria \\
\hline$\sigma$ & Cuidados de eliminación, sondas y drenajes \\
\hline$\sigma$ & $\begin{array}{l}\text { Cuidad. de higiene y prevención de la } \\
\text { infección }\end{array}$ \\
\hline$\infty$ & Medicación \\
\hline 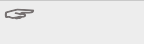 & Cuidados de movilización \\
\hline$\sigma$ & Obtención de muestras \\
\hline$\sigma$ & Cuidados de oxigenación \\
\hline$\sigma$ & Procedimientos Diagnósticos y terapéuticos \\
\hline$\sigma$ & Cuidados de seguridad \\
\hline$\sigma$ & Urgencias \\
\hline$\sigma$ & Vendaje compresivo \\
\hline
\end{tabular}

El objetivo de este trabajo fue describir la puesta en marcha y organización de la Consulta de Enfermería Vascular del Hospital Universitario Vall d'Hebron, detallar la actividad realizada durante sus primeros seis meses de existencia, analizar los obstáculos que dificultan la práctica profesional autónoma de las enfermeras que trabajan en esa consulta y proponer soluciones. 


\section{Metodología}

Tras una exhaustiva revisión y lectura de artículos de revistas especializadas en cirugía vascular, de la Guía de Práctica Clínica de la Asociación Española de Enfermería Vascular (15) y de otros artículos relacionados con consultas de enfermería en hospitales de referencia, en septiembre de 2019 se inició el proyecto para la implantación de la Consulta de Enfermería Vascular. Una vez consensuado el proyecto con todos los miembros del equipo, se decidió la apertura de la consulta a nivel físico e informático.

Con el fin de poder contar con una agenda propia, los responsables de enfermería de las consultas externas solicitaron a los servicios informáticos del hospital añadir una consulta más al listado ya existente de las consultas de los médicos del Servicio de ACV. A la consulta se le asignó el nombre de "Consulta Enfermería Vascular" (CEV), un número de despacho para su ubicación física, y se destinaron tres enfermeras para trabajar en turno de mañana, de lunes a viernes. Respecto a la organización, la agenda CEV se generó a partir de otra agenda denominada "Pendientes de cita", gestionada por el personal administrativo de consultas externas, en la que se iban incluyendo las peticiones de visita solicitadas por los cirujanos o por las propias enfermeras.

A partir de la comunicación in situ entre cirujanos y enfermeras $y$, utilizando como guía el documento de la sociedad española de medicina de familia y comunitaria (semFYC) de 2012 (5), se describió y clasificó el perfil del paciente candidato a ser atendido en la CEV en base a tres grandes grupos: enfermedad arterial periférica (EAP), insuficiencia venosa crónica (IVC) y pie diabético (PD). Posteriormente, se diseñaron planes de cuidados estandarizados para estos grupos de pacientes a partir de la interrelación NANDA/NOC/NIC (16).

En cada primera visita las enfermeras realizan una valoración integral focalizada de los paciente que les permite individualizar estos planes de cuidados estandarizados, adecuándolos a las necesidades de cuidados concretas de cada persona.

Como SAP no permite emplear vocabularios enfermeros normalizados, se creó una base de datos (una plantilla de Excel no integrada en HC3) donde conservar la información relativa a los juicios clínicos (diagnósticos NANDA), a los resultados de salud esperados (criterios de resultado NOC) y a las actuaciones realizadas o planificadas (intervenciones NIC). Para que los datos (prestaciones de cuidados) registrados en SAP reflejasen de la mejor forma posible la naturaleza de las actuaciones realizadas en cada visita, se decidió vincular (de forma consensuada entre las enfermeras) las intervenciones NIC más habituales con las prestaciones disponibles en SAP. Por ejemplo, para dejar constancia de las intervenciones orientadas al fomento del ejercicio o al manejo de energía realizadas en un paciente con Deterioro de la deambulación (NANDA 00085), la prestación de cuidados seleccionada será "Cuidados de movilización", y en el caso de los pacientes que van a ser intervenidos y presentan Temor (NANDA 00148), la prestación de cuidos que se registra en SAP tras realizar intervenciones de apoyo emocional o de disminución de la ansiedad será "Cuidados de comunicación e interacción".

Con el fin de obtener información sobre el tipo de curas realizadas en la CEV también se registraron diariamente el tipo de heridas que presentaban los pacientes atendidos. Las curas se clasificaban según fuesen: úlceras de extremidades inferiores (EEII), heridas quirúrgicas (tras cirugía convencional o endovascular) y esclerosis venosas.

Por último, aunque en la CEV no se emplea la terminología ATIC, para realizar este estudio se realizó una somera valoración de su posible utilidad en nuestro contexto clínico. Para ello se consultó el mapeo del eje diagnóstico ATIC a la taxonomía NANDA que realizó Juvè i Udina para examinar la validez de criterio de ATIC, y se extrajo un listado que recoge algunos de los problemas más frecuentes entre los pacientes de la CEV. Dichos problemas aparecen expresados en ambos vocabularios (ATIC y NANDA). La autora de esta terminología estableció las equivalencias teniendo en cuenta el significado (definición) de los diagnósticos, no su denominación (etiqueta diagnóstica).

Este estudio analiza las prestaciones de cuidados, Ilevadas a cabo en la CEV del Hospital Universitario Vall d'Hebron, desde septiembre de 2019 a marzo de 2020.

Las variables incluidas en el estudio fueron: edad, sexo, número de visitas de la consulta, diagnóstico médico principal, diagnósticos enfermeros, prestaciones enfermeras ligadas a la visita y tipo de heridas que presentaba el paciente. Los datos sociodemográficos, el número de visitas, el diagnóstico médicos y las prestaciones fueron extraídos de manera anonimizada a través de la HCE. Por otra parte, mediante la revisión de los datos recogidos en la tabla de Excel se pudo estimar cuáles eran los diagnósticos de enfermería más prevalentes entre las diferentes tipologías de pacientes.

\section{Resultados}

Durante el periodo comprendido entre el 16 de septiembre de 2019 y el 15 de marzo de 2020, en la CEV del Hospital Vall d'Hebron se realizaron 264 visitas de enfermería, generadas por la atención de 153 pacientes.

De los 153 pacientes estudiados, 77 fueron mujeres $(50,33 \%)$ y 76 hombres $(49,67 \%)$. La Figura 1 recoge la distribución de pacientes por edad. 


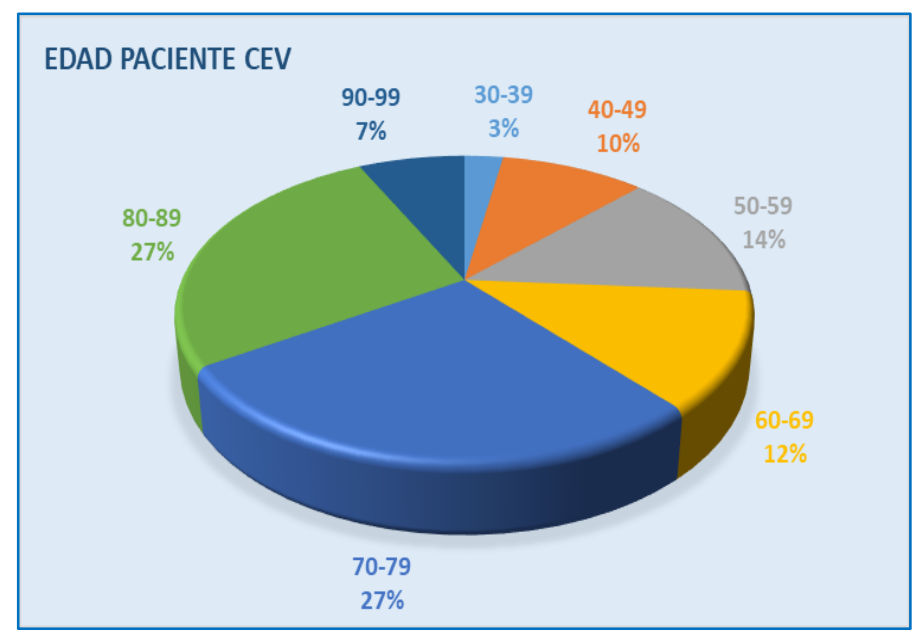

Fig. 1: Distribución de los pacientes según edad ( $n=153)$

Al relacionar edad y sexo, se obtuvo una pirámide bastante homogénea, con un ligero aumento del género masculino a partir de los 70 años (Figura 2).

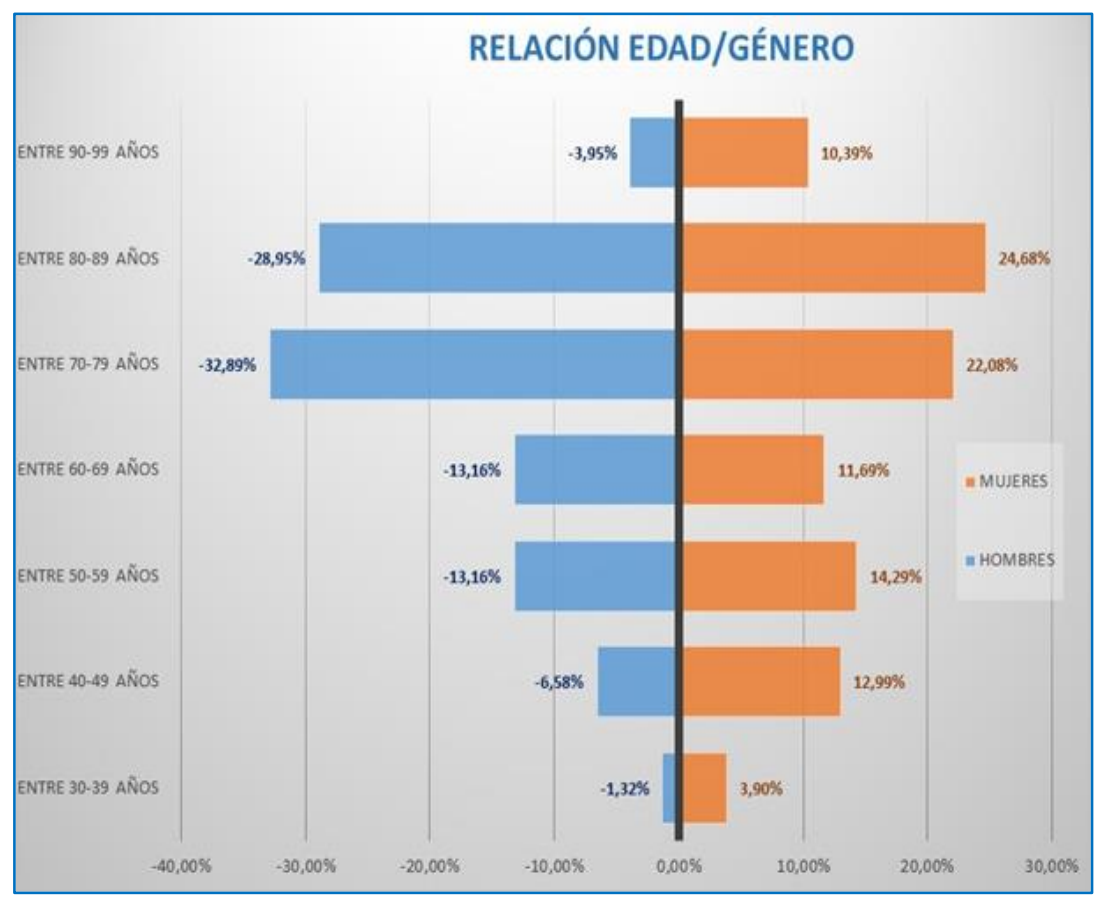

Fig. 2: Distribución de los pacientes según edad y sexo ( $n=153)$

Tal y como se observa en la Figura 3, durante el periodo estudiado, los diagnósticos médicos principales introducidos en el sistema fueron EAP e IVC.

\section{Diagnósticos principales SAP}

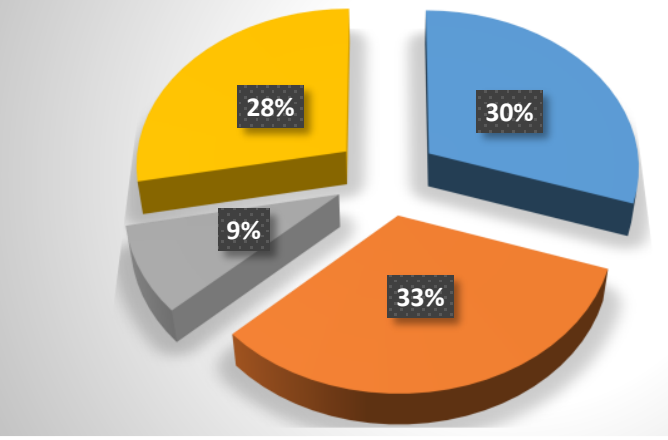


La mayor parte de las curas realizadas correspondieron a úlceras de las EEll (Figura 4).

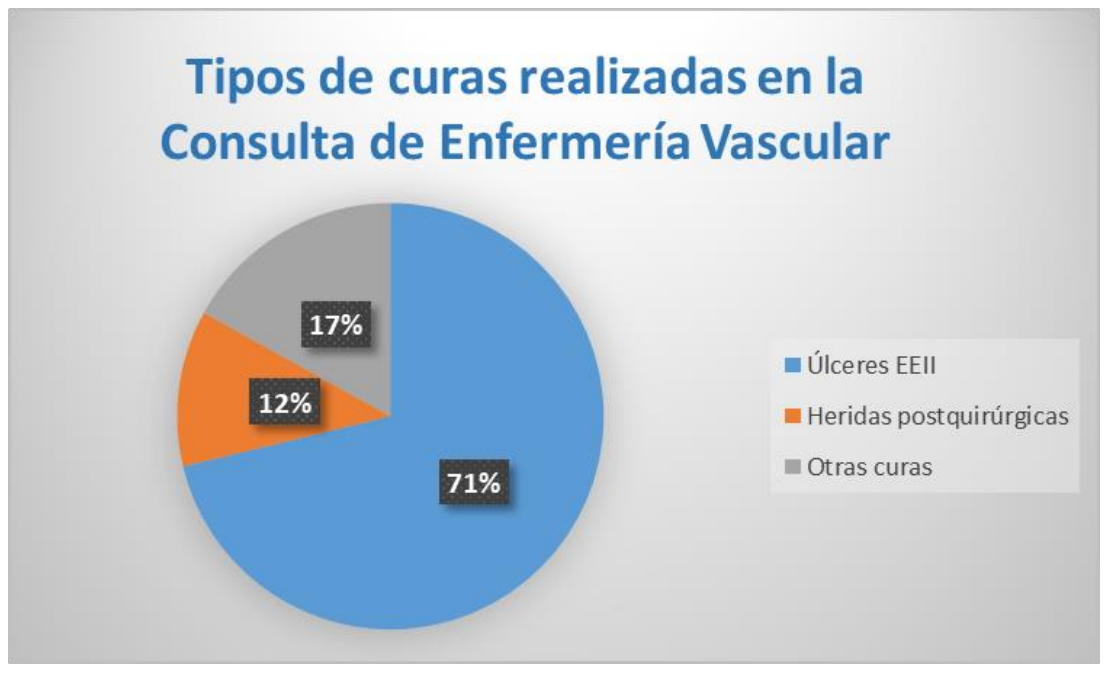

Fig. 4: Curas más frecuentes realizadas en la CEV

Por otro lado, las prestaciones enfermeras ligadas a las visitas y registradas con más frecuencia en el aplicativo, fueron Educación sanitaria y Cuidados de heridas y otras lesiones (Figura 5).

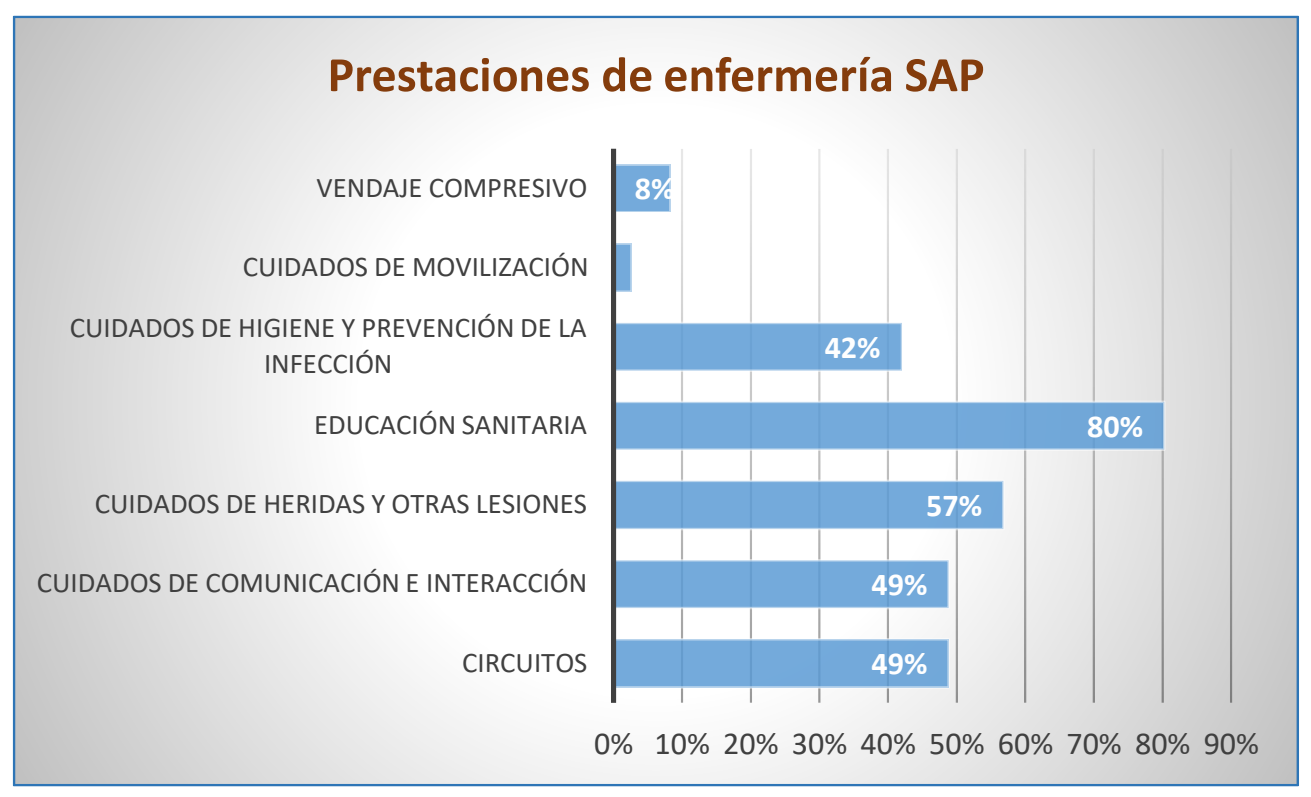

Fig. 5: Prestaciones de enfermería más habituales

Entre los pacientes del grupo Enfermedad Arterial Periférica, los diagnósticos NANDA más frecuentes fueron: Dolor agudo (00132), Dolor crónico (00133), Perfusión tisular periférica ineficaz (00204), Riesgo de disfunción neurovascular periférica (00086), Deterioro de la integridad cutánea (00046) y Riesgo de infección (00004).

En el grupo Insuficiencia Venosa Crónica, los diagnósticos NANDA más observados fueron: Riesgo de infección (00004), Deterioro de la integridad cutánea (00046) y Perfusión tisular periférica ineficaz (00204).
Entre los pacientes pertenecientes al grupo Pie Diabético, los diagnósticos NANDA más frecuentes fueron: Deterioro de la integridad cutánea (00046), Perfusión tisular periférica ineficaz (00204), Deterioro de la deambulación (00088), Riesgo de disfunción neurovascular periférica (00086), Trastorno de la imagen corporal (00118), Dolor agudo (00132), Riesgo de infección (00004), Afrontamiento inefectivo (0069), Dolor crónico (00133) y Duelo anticipado (00136).

La Tabla 2 recoge los problemas más habituales entre los pacientes de la CEV expresados en terminología ATIC y su equivalencia con diagnósticos NANDA. 
Tabla 2: Correlación terminología ATIC / diagnósticos NANDA

\begin{tabular}{|c|c|c|}
\hline ATIC 2012-2017 & TIPO & NANDA 2015-2017 \\
\hline $\begin{array}{l}\text { Deterioro de la adaptación al nuevo estado de } \\
\text { salud }\end{array}$ & $1: A$ & Gestión ineficaz de la salud \\
\hline Ansiedad & $1: 1$ & Ansiedad \\
\hline Ansiedad preoperatoria & $1: A$ & Ansiedad \\
\hline Ansiedad ante la muerte & $1: 1$ & Ansiedad \\
\hline Déficit de autocuidado: higiene & $1: 1$ & Déficit de autocuidado: baño \\
\hline Déficit de autocuidado: Alimentación & $1: 1$ & Déficit de autocuidado: alimentación \\
\hline Déficit de autocuidado: vestir & $1: 1$ & Déficit de autocuidado: vestido \\
\hline Baja autoestima crónica & $1: 1$ & Baja autoestima crónica \\
\hline Riesgo de baja autoestima crónica & $1: 1$ & Riesgo de baja autoestima crónica \\
\hline Baja autoestima crónica familiar & $1: A$ & Baja autoestima crónica \\
\hline Riesgo de baja autoestima crónica familiar & $1: \mathrm{A}$ & Riesgo de baja autoestima crónica \\
\hline Baja autoestima crónica parental & $1: \mathrm{A}$ & Baja autoestima crónica \\
\hline Riesgo de baja autoestima crónica parental & $1: A$ & Riesgo de baja autoestima crónica \\
\hline Baja autoestima situacional & $1: 1$ & Baja autoestima situacional \\
\hline Riesgo de baja autoestima situacional & $1: 1$ & Riesgo de baja autoestima situacional \\
\hline Baja autoestima situacional familiar & $1: \mathrm{A}$ & Baja autoestima situacional \\
\hline Riesgo de baja autoestima situacional & $1: A$ & Riesgo de baja autoestima situacional \\
\hline Riesgo de autoexclusión involuntaria & $1: \mathrm{A}$ & Deterioro de la interacción social \\
\hline Riego de autoexclusión protectora, & $1: \mathrm{A}$ & Deterioro de la interacción social \\
\hline Riesgo de caída & $1: N$ & Riesgo de caídas \\
\hline Dinon do laudingión namotal & & Cansancio del rol de cuidador \\
\hline Riesgo de clauaicacion parental & $1: \mathrm{N}$ & Afrontamiento familiar comprometido \\
\hline Déficit nutricional & $1: A$ & $\begin{array}{l}\text { Desequilibrio nutricional: inferior a las necesidades } \\
\text { corporales }\end{array}$ \\
\hline Descamación cutánea & $1: A$ & Deterioro de la integridad cutánea \\
\hline Descamación húmeda & $1: \mathrm{A}$ & Deterioro de la integridad tisular \\
\hline Desesperanza & $1: 1$ & Desesperanza \\
\hline Deterioro neuromuscular crónico & $1: \mathrm{A}$ & Deterioro de la movilidad física \\
\hline Deterioro de la Dinámica familiar & $1: 1$ & Procesos familiares disfuncionales \\
\hline Riesgo de deterioro de la Dinámica familiar & $1: 0$ & \\
\hline Dolor & $1: 1$ & Dolor \\
\hline Dolor neuropático & $1: \mathrm{A}$ & Dolor agudo \\
\hline Dolor postoperatorio & $1: \mathrm{A}$ & Dolor agudo \\
\hline Duelo & $1: 1$ & Duelo \\
\hline Duelo anticipado & $1: \mathrm{A}$ & Duelo \\
\hline Duelo complicado & $1: 1$ & Duelo complicado \\
\hline Edema & $1: A$ & Exceso de volumen de líquidos \\
\hline Edema periférico & $1: 0$ & \\
\hline Erosión cutánea & $1: \mathrm{A}$ & Deterioro de la integridad cutánea \\
\hline Estreñimiento & $1: 1$ & Estreñimiento \\
\hline Flebitis & $1: 0$ & \\
\hline Hematoma & $1: 0$ & \\
\hline Hemorragia & $1: 0$ & \\
\hline Riesgo de Hemorragia & $1: 1$ & Riesgo de sangrado \\
\hline $\begin{array}{l}\text { Riesgo de recurrencia/ progresión de la } \\
\text { Hemorragia }\end{array}$ & $1: 0$ & \\
\hline Herida Contaminada & $1: \mathrm{A}$ & Deterioro de la integridad cutánea \\
\hline Herida infectada & $1: \mathrm{A}$ & Deterioro de la integridad tisular \\
\hline
\end{tabular}


Tabla 2: Continuación

\begin{tabular}{|c|c|c|}
\hline ATIC 2012-2017 & TIPO & NANDA 2015-2017 \\
\hline Herida limpia & $1: A$ & Deterioro de la integridad cutánea \\
\hline Herida quirúrgica & $1: A$ & Deterioro de la integridad tisular \\
\hline Herida quirúrgica contaminada & $1: A$ & Deterioro de la integridad tisular \\
\hline Herida quirúrgica infectada & $1: \mathrm{A}$ & Deterioro de la integridad tisular \\
\hline Herida quirúrgica limpia & $1: \mathrm{A}$ & Deterioro de la integridad tisular \\
\hline Riesgo de Hiper/Hipoglucemia & $1: 1$ & Riesgo de nivel de glicemia inestable \\
\hline Trastorno de la imagen corporal & $1: 1$ & Trastorno de la imagen corporal \\
\hline Impotencia funcional & $1: \mathrm{A}$ & Deterioro de la movilidad física \\
\hline Incumplimiento del tratamiento & $1: 1$ & Incumplimiento \\
\hline Riesgo de incumplimiento del tratamiento & $1: A$ & Mantenimiento ineficaz de la salud \\
\hline Riesgo de infección & $1: 1$ & Riesgo de infección \\
\hline Riesgo de infección posoperatoria & $1: A$ & Riesgo de infección \\
\hline Injerto cutáneo & $1: 0$ & \\
\hline Riesgo de isquemia & $1: \mathrm{N}$ & $\begin{array}{l}\text { Riesgo de disminución de la perfusión tisular } \\
\text { cardiaca; cerebral, renal, periférica }\end{array}$ \\
\hline Lesión cutánea infecciosa & $1: 0$ & \\
\hline Lesión dermatoporótica grado I & 1: A & Deterioro de la integridad cutánea \\
\hline Lesión dermatoporótica grado II & 1: A & Deterioro de la integridad tisular \\
\hline Lesión dermatoporótica grado III & 1: A & Deterioro de la integridad tisular \\
\hline Lesión dermatoporótica grado VI & 1: A & Deterioro de la integridad tisular \\
\hline Maceración cutánea & 1: A & Deterioro de la integridad cutánea \\
\hline Marcha inestable & 1: A & Deterioro de la deambulación \\
\hline $\begin{array}{l}\text { Riesgo de manejo inefectivo del régimen } \\
\text { terapéutico }\end{array}$ & 1: A & Gestión ineficaz de la salud \\
\hline Muñón & $1: 0$ & \\
\hline Obesidad & $1: 1$ & Obesidad \\
\hline Riesgo de obesidad & $1: 0$ & \\
\hline prurito & $1: A$ & disconfort \\
\hline Síndrome ansioso depresivo & $1: 0$ & \\
\hline Riesgo de síndrome ansioso depresivo & $1: E$ & Ansiedad \\
\hline Síndrome de déficit de autocuidado & $1: \mathrm{N}$ & $\begin{array}{l}\text { Déficit de autocuidado, alimentación; baño; uso del } \\
\text { inodoro; vestido }\end{array}$ \\
\hline Riesgo de síndrome de robo arterial & $1: A$ & Riesgo de perfusión tisular periférica ineficaz \\
\hline Síndrome del miembro fantasma & $1: 0$ & \\
\hline sobrepeso & $1: 1$ & sobrepeso \\
\hline Sufrimiento espiritual & $1: 1$ & Sufrimiento espiritual \\
\hline Temor a las caídas & $1: A$ & Temor \\
\hline Riesgo de tromboembolismo & $1: \mathrm{N}$ & $\begin{array}{l}\text { Riesgo de perfusión tisular cerebral ineficaz; } \\
\text { gastrointestinal; renal; cardiaca, periférica }\end{array}$ \\
\hline $\begin{array}{l}\text { Riesgo de recurrencia/progresión del } \\
\text { tromboembolismo }\end{array}$ & $1: 0$ & \\
\hline
\end{tabular}


Tabla 2: Continuación

\begin{tabular}{|c|c|c|}
\hline ATIC 2012-2017 & TIPO & NANDA 2015-2017 \\
\hline Riesgo de trombosis venosa profunda & $1: A$ & Riesgo de perfusión tisular ineficaz \\
\hline Úlcera & $1: A$ & Deterioro de la integridad tisular \\
\hline Ulcera arterial & $1: \mathrm{A}$ & Deterioro de la integridad tisular \\
\hline Ulcera pie diabético & $1: \mathrm{A}$ & Deterioro de la integridad tisular \\
\hline Ulcera pie diabético & $1: A$ & Deterioro de la integridad tisular \\
\hline Ulcera pie diabético grado I & $1: A$ & Deterioro de la integridad tisular \\
\hline Ulcera pie diabético grado II & $1: A$ & Deterioro de la integridad tisular \\
\hline Ulcera pie diabético grado III & $1: A$ & Deterioro de la integridad tisular \\
\hline Ulcera mixta & $1: \mathrm{A}$ & Deterioro de la integridad tisular \\
\hline Ulcera por presión grado I & $1: A$ & Deterioro de la integridad cutánea \\
\hline Ulcera por presión grado II & $1: \mathrm{A}$ & Deterioro de la integridad cutánea \\
\hline Ulcera por presión grado III & $1: A$ & Deterioro de la integridad cutánea \\
\hline Ulcera por presión grado IV & $1: A$ & Deterioro de la integridad cutánea \\
\hline Riesgo alto de ulcera por presión & $1: A$ & Riesgo de úlcera por presión \\
\hline Riesgo medio de úlcera por presión & $1: A$ & Riesgo de úlcera por presión \\
\hline Riesgo bajo de úlcera por presión & $1: A$ & Riesgo de úlcera por presión \\
\hline Ulcera venosa & $1: A$ & Deterioro de la integridad tisular \\
\hline Uña encarnada & $1: A$ & Deterioro de la integridad tisular \\
\hline Zona dadora & $1: A$ & Deterioro de la integridad tisular. \\
\hline
\end{tabular}

a 1: 1 Concepto en ATIC que tiene un equivalente conceptual idéntico en NANDA.

b 1: 0 Concepto en ATIC que no tiene un equivalente conceptual idéntico en NANDA.

c 1: A Concepto en ATIC con un equivalente conceptual más abstracto en NANDA

d 1: E Concepto en ATIC que tiene un equivalente conceptual más específico en NANDA

e 1: N Concepto en ATIC que tiene múltiples equivalentes conceptuales generalmente más específicos en NANDA

Fuente: Juvé i Udina M.E. La terminología ATIC. Eje diagnóstico. Barcelona: NAAXPOT S.L.U.; 2016. Con permiso de la autora.

\section{Discusión}

Las enfermeras de la CEV del HUVH atienden heridas crónicas tales como úlceras de EEll o úlceras del pie diabético, pero a diferencia de otras experiencias consultadas $(16,17)$, no tratan pacientes con úlceras por presión, por ejemplo, porque en el HUVH estos casos son derivados a la unidad de heridas. También, a diferencia de otras unidades multidisciplinares, en la CEV se realizan curas de heridas quirúrgicas con o sin complicaciones en su cicatrización. Finalmente, otra actuación habitual en la consulta es la colaboración con los médicos en esclerosis venosas, rol de colaboración que, a priori, se consideraba puntual o esporádico, pero que constituye el $17 \%$ de las curas registradas.
Pero el hecho más destacable es que, tal y como se preveía, las enfermeras de la CEV han ganado en autonomía y han pasado de tener un rol meramente colaborador en el tratamiento de problemas interdependientes que diagnostican los cirujanos a tratar y solucionar problemas que ellas mismas diagnostican: las enfermeras de la CEV del HUVH no contemplan únicamente la enfermedad y no solo curan heridas; las enfermeras de la CEV ofrecen cuidados integrales dentro de un contexto multidisciplinar, tanto a pacientes agudos como a pacientes crónicos, tanto a pacientes con úlceras en EEII como a pacientes quirúrgicos y, por lo tanto, aparte de realizar curas y pruebas diagnósticas o de colaborar en ciertos procedimientos terapéuticos, 
sus funciones incluyen actuaciones destinadas a la promoción de la salud, a la prevención de complicaciones $y$, en general, a mejorar los resultados de salud de los pacientes vasculares. Este escenario implica que las enfermeras al frente de esta consulta necesitan un alto nivel competencial y la capacidad de adaptarse a un perfil de paciente muy heterogéneo.

Por todo lo expuesto, se considera que la actividad descrita de la CEV estaría más relacionada con la de una consulta-clínica de enfermería, tal como la define Pimentel Jaimes (1) que con la de una unidad de heridas. Una unidad de heridas, en la mayoría de los trabajos consultados $(2,3,4,6)$, se define como una estructura que, entre otras, tiene funciones de asesoramiento a otros servicios y un plan de formación específico para el tratamiento de heridas crónicas para otros profesionales del hospital o centro de salud que lo requieran. No es el caso de la CEV del HUVH.

Por otro lado, se observa un hecho diferencial destacable: la organización diseñada para la CEV "blinda" el acceso a la consulta desde otros servicios o unidades que no sean exclusivamente las consultas externas de ACV. A pesar de que, en algunos casos, las curas no sean planificadas y tengan que realizarse en la misma consulta, en la mayoría de los casos el circuito diseñado garantiza que solo los cirujanos o las propias enfermeras de la CEV puedan derivar pacientes a la CEV. Así se puede mantener una actividad sin apenas visitas inesperadas que distorsionen el tiempo dedicado a cada paciente.

Aunque todos los pacientes han sido valorados en la CEV por las enfermeras, al principio, por falta de pericia, era necesario consultar con los cirujanos de ACV o con la enfermera referente de la Unidad de Heridas de nuestro Hospital. También, en ocasiones fue necesaria la comunicación con profesionales de Atención Primaria. Tras los primeros seis meses de funcionamiento se comprobó que muchas problemas se solucionan sin necesidad de que intervengan otros profesionales. Así, se simplifican los procesos asistenciales del paciente, que es atendido en un único espacio físico y sin que tenga que desplazarse a otros servicios o unidades.

El principal obstáculo detectado hasta la fecha es de índole tecnológico, ya que la aplicación informática utilizada en las consultas externas del hospital, lejos de facilitar el rol autónomo enfermero, lo dificulta. Por un lado, al no tratarse de una herramienta pensada para implementar el proceso enfermero, SAP impide la valoración de las actuaciones realizadas. Por otro lado, en vez de permitir el uso de lenguajes normalizados, SAP obliga a elegir entre un listado de actuaciones (Figura 1) en el que se mezclan unos términos de fácil comprensión para cualquier profesional (como por ejemplo Vendaje compresivo o Educación sanitaria) con otros excesivamente abstractos (Cuidados de comunicación e interacción) y con alguno que, seguramente, escapa al entendimiento de cualquier profesinal que no trabaje en el HUVH y cuyo registro tampoco aporta valor a lo que se considera práctica autónoma centrada en resultados del paciente, como es el caso, por ejemplo, de la prestación enfermera denominada Circuitos.

La detección de este obstáculo está en consonancia con lo expuesto por Juvé i Udina (9), cuando sostiene que, a pesar de que los cuidados enfermeros contribuyen a salvar vidas, a promover y a recuperar el estado de salud, a aliviar el sufrimiento, a potenciar la autonomía, y aunque ello requiere una formación sólida y un desarrollo competencial continuado, en la mayoría de ocasiones los servicios enfermeros no se ven reflejados en las bases de datos que apoyan la toma de decisiones de las empresas de salud. En ausencia de datos, la generación de información sobre la naturaleza, la efectividad y el impacto de los servicios que prestan las enfermeras es inviable, cronificando su invisibilidad.

Independientemente de similitudes y diferencias con otras unidades o consultas, pensamos que, en este tipo de consultas donde es tan importante la comunicación con otros miembros del equipo multidisciplinar para garantizar la continuidad de cuidados, sería especialmente beneficioso el empleo de una terminología de entrada que, además de aportar valor a los registros, sea fácilmente comprensible por otros profesionales. Ese es el caso de ATIC, que, además de cumplir estos requisitos, cuenta con la ventaja adicional de incorporar unos descriptores del estado del paciente y de las actuaciones de las enfermeras que son muy específicos. Como ejemplo de esto, basta con comprobar que, frente a una única etiqueta diagnóstica NANDA como Deterioro de la integridad tisular, ATIC permite elegir entre una gran variedad de términos diferentes (Tabla 2). De igual forma, ATIC permite denominar y registrar algunos conceptos que no tienen un equivalente en NANDA como son Flebitis, Hematomas o Hemorragia, problemas que son observados con frecuencia en los pacientes que acuden a las consultas externas de ACV.

Hasta hace poco, la enfermera de consultas externas desarrollaba su actividad centrándose prácticamente en los aspectos administrativos y de colaboración con otros profesionales. Con la puesta en marcha de la CEV la actividad enfermera se lleva a cabo en un despacho concreto y es la enfermera quien gestiona su agenda, de manera que puede proporcionar cuidados en su concepto más amplio.

La integración de una Consulta de Enfermería en las consultas externas del Servicio de ACV nos lleva a plantearnos futuras líneas de investigación: 
¿Cuál es el grado de satisfacción de los pacientes, de las enfermeras y de los médicos especialistas con respecto a la integración de la CEV en las consultas externas de ACV? ¿Reúnen las enfermeras de la CEV las características del perfil de la Enfermera de Práctica Avanzada descritas por el Consejo Internacional de Enfermeras (CIE)? En relación a las nuevas tecnologías/prestaciones: ¿Puede una llamada telefónica sustituir, en algunos casos, la visita presencial del paciente? ¿Es necesaria la gestión de imágenes vía telemática para el correcto seguimiento de las úlceras de EEII? Sin duda se abre un amplio campo de investigación en el que no puede faltar el uso de las nuevas tecnologías y la adaptación continua a las demandas y necesidades de los usuarios.

\section{Conclusiones}

La Consulta de Enfermería Vascular potencia la autonomía de la práctica profesional y favorece la atención personalizada a los pacientes de las consultas externas del Servicio de Angiología y Cirugía Vascular. Para que las enfermeras de esta consulta puedan desarrollar todo su potencial, y para que los resultados de sus actuaciones sean mesurables y visibles, es necesario implementar sistemas de información asistencial que sean compatibles con una herramienta metodológica tan básica para la profesión como es el proceso enfermero, y que permitan registrar los datos derivados de la valoración del paciente y de toda la actividad realizada mediante vocabularios normalizados. Dentro de los vocabularios empleados en nuestro entorno, pensamos que la terminología ATIC sería la opción más conveniente.

\section{Conflicto de intereses:}

Ninguno.

\section{Bibliografía}

1. Pimentel-Jaimes JA, Casique-Casique L, ÁlvarezAguirre A, Higuera-Sainz'L, Bautista-Alvarez TM. Consulta de enfermería: un análisis de concepto. sanus [Internet]. 30 de junio de 2019 [Consultado 10 de mayo de 2020] ;(10):70-4. Disponible en: https://sanus.unison.mx/index.php/Sanus/article/ view/134.

2. Esparza G, Fuentes A, Morales MJ, Nova JM. Visión y experiencia de enfermeras coordinadoras de unidades de heridas crónicas. Gerokomos. 2016;27 (3):127-30.

3. González de la Torre Héctor, Verdú Soriano José, García Fernández Francisco Pedro, Soldevilla Ágreda Javier. Primer censo de unidades de heridas crónicas en España. Gerokomos. 2017;28(3):14250.
4. García-Fernández, FP; López-Casanova, P; SegoviaGómez, T; Soldevilla-Agreda, JJ; Verdú-Soriano, J. Unidades Multidisciplinares de Heridas Crónicas: Clínicas de Heridas. Serie Documentos de Posicionamiento GNEAUPP no 10. Grupo Nacional para el Estudio y Asesoramiento en Úlceras por Presión y Heridas Crónicas. Logroño. 2012. [Consultado el 9 de mayo de 2020]. Disponible en:

https://gneaupp.info/wp-content/uploads/2014/12/ unidades-multidisciplinares-de-heridas-cronicas.pdf

5. Bellmunt Montoya $\mathrm{S}$, et al. Criterios de derivación entre niveles asistenciales de pacientes con patología vascular. Documento de consenso semFYCSEACV. Aten Primaria. 2012. 64(3): 135-45.

6. Hernández Bezos, A. Beneficios de las Unidades Multidisciplinares en el abordaje de las Heridas Crónicas. Revisión Bibliográfica. [Consultado el 7 de mayo de 2020]. Disponible en:

http://uvadoc.uva.es/bitstream/10324/31683/1/TFG -L2105.pdf

7. Luis Rodrigo MT, Fernández Ferrín C, Navarro Gómez MV. De la teoría a la práctica. El pensamiento de Virginia Henderson en el siglo XXI. 3. a ed. BarceIona: Elsevier Masson; 2005.

8. Alfaro-Lefevre, R. Aplicación del proceso enfermero. Guía paso a paso. 3. a ed. Barcelona: Springer-Verlag Iberica; 1999.

9. Juvé i Udina ME. Evaluación inductiva de la estructura de una terminología enfermera de interfase: conceptualización del proceso enfermero. Nursing (Ed. española). 2012; 30(7): 62-6. doi: 10.1016/ S0212-5382(12)70109-7

10. NANDA diagnósticos enfermeros: definiciones y clasificación 2018-2020.11.a ed. Barcelona: Elsevier España.S.L.U; 2019.

11. Butcher HK, Bulechek GM,, Dochterman JM, Wagner CM. Clasificación de intervenciones de enfermería (NIC). 7. a ed. Elsevier; 2018.

12. Moorhead S, Swanson E, Johnson $M$, Maas $M$. Clasificación de Resultados de Enfermería (NOC). Medición de Resultados en Salud. 6. $\underline{\text { a ed. Elsevier; }}$ 2019.

13. Juvé i Udina ME. La terminología ATIC. Eje diagnóstico. Barcelona: NAAXPOT S.L.U.; 2016.

14. Web Departament de Salut de Catalunya [nternet] [consultado el 10/06/2020]. Disponible en: https://salutweb.gencat.cat/ca/ambits_tematics/li nies_dactuacio/tecnologies_informacio_i_comunic acio/historia_clinica_compartida/

15. Asociación Española de Enfermería Vascular y Heridas. Guía de práctica clínica: Consenso sobre úlce-ras vasculares y pie diabético. Tercera edición. Ma-drid: AEEVH, 2017. [Internet]. 2019 [Consultado el 10 de mayo de 2020]. Disponible en: https://www.aeev.net/ 
16. Herramienta online para la consulta y diseño de Planes de Cuidados de enfermería. [Internet]. NNN Consult. Elsevier: 2015 [Consultado el 10 de mayo de 2020]. Disponible en: https://www.nnnconsult.com/

17. Juvé Udina ME, González Samartino $M$, Matud Calvo C. Mapeo del eje de diagnóstico de una terminología de interfaz a la taxonomía inter-nacional NANDA. ISRN Enfermera. 2012; 2012: 676905. doi: 10.5402 / 2012/676905.
18. Scarborough P. Understanding Your Wound Care Team. Defining Unidisciplinary, MultIdisciplinary, Interdisciplinary and Transdisciplinary Team Models. [Acceso 19 de mayo de 2020]. Disponible en: http:// www.woundsource.com/whitepaper/understanding-your-wound-care-team

19. Rondas AA, Schols JM, Halfens RJ, Hull HR, Stobberingh EE, Evers SM. Cost analysis of one of the first outpatient wound clinics in the Netherlands. J Wound Care. 2015;24(9):426-36. 